\title{
WHAT CONTROLS THE STAR FORMATION IN LUMINOUS STARBURST MERGERS ?
}

\author{
Yoshiaki Taniguchi and Youichi Ohyama \\ Astronomical Institute, Tohoku University, Aoba, Sendai 980-8578, Japan
}

Received — 


\begin{abstract}
In order to understand what controls the star formation process in luminous starburst mergers (e.g., NGC 6240, Arp 220, and so on), we investigate observational properties of two samples of high-luminosity starburst galaxies mapped in $\mathrm{CO}(J=1-0)$ independently using both the Owens Valley Radio Observatory (Scoville et al. 1991) and the IRAM interferometer (Downes \& Solomon 1998). We find that the surface density of far-infrared luminosity, $\Sigma(\mathrm{FIR})$, is proportional linearly to the $\mathrm{H}_{2}$ surface mass density, $\Sigma\left(\mathrm{H}_{2}\right)$, for the two samples; $\Sigma(\mathrm{FIR}) \propto \Sigma\left(\mathrm{H}_{2}\right)^{1.01 \pm 0.06}$ with a correlation coefficient of 0.96 . It is often considered that $\Sigma(\mathrm{FIR})$ provides a good measure of the star formation rate per unit area, $\Sigma(\mathrm{SFR})$. It is also known that molecular gas is dominated in circumnuclear regions in the luminous starburst mergers; i.e., $\Sigma($ gas $) \simeq \Sigma\left(\mathrm{H}_{2}\right)$. Therefore, the above relationship suggests a star formation law; $\Sigma(\mathrm{SFR})$ $\propto \Sigma$ (gas). We suggest that this star formation law favors the gravitational instability scenario rather than the cloud-cloud collision one.
\end{abstract}

Subject headings: galaxies: starburst - ISM: molecules - stars: formation 


\section{INTRODUCTION}

Since the discovery of ultraluminous infrared galaxies (ULIGs: Soifer et al. 1984; Wright, Joseph, \& Meikle 1984; see for a review Sanders \& Mirabel 1996), much attention has been paid to this class of galaxies (hereafter starburst mergers[). Most of these galaxies show morphological evidence for galaxy mergers or strong galaxy interaction. There is still a controversy on the origin of their huge infrared luminosities; i.e., a pure starburst or a hidden central engine of active galactic nuclei (AGN). Recently, growing evidence has been accumulated for the relative importance of starbursts in their central regions (e.g., Shaya et al. 1994; Genzel et al. 1998; Taniguchi, Trentham, \& Shioya 1998 and references therein). However, an important question still remains; what controls the star formation process in the starburst mergers? Therefore, it is very important to investigate this problem.

A number of previous $\mathrm{CO}$ observations have shown that the starburst mergers have a lot of molecular gas up to $\sim 10^{10} M_{\odot}$ (Young et al. 1986a, 1986b; Sanders et al. 1988; Solomon \& Sage 1988; Sanders, Scoville, \& Soifer 1991). Moreover, the molecular gas is usually distributed in the inner $1 \mathrm{kpc}$ region in these mergers [Scoville et al. 1991 (hereafter S91); Scoville, Yun, \& Bryant 1997; Downes \& Solomon 1998 (hereafter DS98)]. Therefore, it has been considered that the accumulation of a lot of molecular gas toward the central region can lead to the intense starbursts in the mergers. Indeed, numerical simulations have shown that galaxy mergers can fuel gas clouds toward the central region of the merger products (Barnes \& Hernquist 1991; Mihos \& Hernquist 1994). Here a question arises as how the intense starbursts were triggered in the fueled gas. There may be three alternative basic physical processes to cause the intense starbursts; 1) the gravitational instability in nuclear gas disks (e.g., Kennicutt 1998 and references therein), 2) efficient

\footnotetext{
${ }^{1}$ In this Letter, we do not use a more popular terminology, ultraluminous infrared galaxies, because our sample studied here contains mergers with $L($ FIR $)<10^{12} L_{\odot}$.
} 
cloud-cloud collision driven by violent gas motion in their central regions (e.g., S91), and 3) triggering through the dynamical disturbance driven by supermassive binary black holes (Taniguchi \& Wada 1996). In order to investigate which process is most important in the starburst mergers, we analyze two data sets of high-luminosity starburst mergers mapped in $\mathrm{CO}(J=1-0)$ using the Owens Valley Radio Observatory (OVRO) interferometer (S91) and the IRAM one (DS98).

\section{DATA}

The first data set is taken from S91 who presented a summary of the OVRO $\mathrm{CO}(J=1-0)$ mapping of 18 high-luminosity infrared galaxies. Almost all the galaxies show evidence either for galaxy mergers or for strong galaxy interaction. This sample covers a wide range of far-infrared (FIR) luminosities from $\sim 10^{10} L_{\odot}$ to $\sim 10^{12} L_{\odot}$. The second data set is taken from DS98 who performed detailed $\mathrm{CO}(J=1-0)$ and $\mathrm{CO}(J=2-1)$ observations of ten ULIGs. Though four ULIGs among this sample are also included in the OVRO sample (Arp 220, Mrk 231, VII Zw 31, and IRAS 17208-0014), we deal with this IRAM sample as an independent sample appreciating the two independent CO mapping programs using the two different radio interferometers. Although these two samples are not statistically complete ones, they are highly useful in investigating a star formation law in starburst mergers. In Table 1, we give a summary of observational properties of the individual galaxies of the two samples. We use the distances of galaxies estimated using both recession velocities corrected to the Galactic Standard of Rest (GSR) following the manner described in de Vaucouleurs et al. (1991) and a Hubble constant $H_{0}=75 \mathrm{~km} \mathrm{~s}^{-1} \mathrm{Mpc}^{-1}$. The heliocentric velocities are taken from de Vaucouleurs et al. (1991), S91, and DS98 (see Table 1).

Molecular gas masses for all the galaxies in the two samples are derived using a conversion factor, $\alpha=N_{\mathrm{H}_{2}} / I(\mathrm{CO})=3 \times 10^{20} \mathrm{~cm}^{-2}\left(\mathrm{~K} \mathrm{~km} \mathrm{~s}^{-1}\right)^{-1}$ where $N_{\mathrm{H}_{2}}$ is the $\mathrm{H}_{2}$ 
column density and $I(\mathrm{CO})$ is the integrated intensity of the $\mathrm{CO}(J=1-0)$ emission (S91). The FIR data are compiled from the IRAS Faint Source Catalog (Moshir et al. 1992). The FIR luminosities are estimated using $L(\mathrm{FIR})=4 \pi D^{2} 1.26 \times 10^{-11}[2.58 \times S(60)+S(100)]$ $\left(\operatorname{ergs~s}{ }^{-1}\right.$ ) where $S(60)$ and $S(100)$ are the IRAS $60 \mu \mathrm{m}$ and $100 \mu \mathrm{m}$ fluxes in units of Jy and $D$ is the distance of galaxies in units of cm (Helou, Soifer, \& Rowan-Robinson 1985). Finally $L(\mathrm{FIR})$ is given in units of $L_{\odot}$. In later analysis, in addition to $L(\mathrm{FIR})$ and $M_{\mathrm{H}_{2}}$, we also use their surface densities; $\Sigma(\mathrm{FIR})\left(L_{\odot} \mathrm{pc}^{-2}\right)$ and $\Sigma\left(\mathrm{H}_{2}\right)\left(M_{\odot} \mathrm{pc}^{-2}\right)$, respectively. These densities for each galaxy are normalized by a surface $A=\pi r^{2}$ where $r$ is the radius in units of pe given in S91 and DS98. Note that for the four ULIGs commonly observed by both S91 and DS98, the CO sizes in DS98 are systematically smaller than those in S91 (see Table 1). We use the CO sizes given in DS98 for these four ULIGs in the later analysis.

\section{RESULTS}

In Figure 1, we show diagrams of $\log L(\mathrm{FIR})\left(L_{\odot}\right), \log \Sigma(\mathrm{FIR})\left(L_{\odot} \mathrm{pc}^{-2}\right)$, and $\log$ $L(\mathrm{FIR}) / M_{\mathrm{H}_{2}}\left(L_{\odot} / M_{\odot}\right)$ as functions of $\log \Sigma\left(\mathrm{H}_{2}\right)\left(M_{\odot} \mathrm{pc}^{-2}\right)$ and $\log S(60) / S(100)$ for the two samples of S91 and DS98. Among the six relationships shown in Figure 1, we find the following two significant correlations for both the samples; a) $\log \Sigma(\mathrm{FIR})$ vs. $\log \Sigma\left(\mathrm{H}_{2}\right)$, and b) $\log L(\mathrm{FIR}) / M_{\text {dust }}$ vs. $\log S(60) / S(100)$.

We obtain the nearly linear relationship between $\Sigma(\mathrm{FIR})$ and $\Sigma\left(\mathrm{H}_{2}\right) ; \Sigma(\mathrm{FIR})$ $\propto \Sigma\left(\mathrm{H}_{2}\right)^{1.02 \pm 0.06}$ for the S91 sample, $\Sigma(\mathrm{FIR}) \propto \Sigma\left(\mathrm{H}_{2}\right)^{1.16 \pm 0.13}$ for the DS98 sample, and

$\Sigma(\mathrm{FIR}) \propto \Sigma\left(\mathrm{H}_{2}\right)^{1.01 \pm 0.06}$ for the total (S91+DS98) sample. Note that the new data in DS98 are adopted for the four common ULIGs in the total sample. Therefore, the total number of ULIGs in the total sample is 24 . The correlation coefficient for the total sample is quite high, $R \simeq 0.96$. Since $\Sigma(\mathrm{FIR})$ can be regarded as the star formation rate (SFR) per unit surface area (e.g., Kennicutt 1998), i.e., $\Sigma($ SFR), this correlation implies that the SFR is 
controlled locally by the surface gas density. On the other hand, since there is no significant correlation between $L(\mathrm{FIR})$ and $\Sigma\left(\mathrm{H}_{2}\right)$, the total FIR luminosity is governed not by the local gas density but by the total gaseous content accumulated in the central region of starburst mergers.

The correlation between $L(\mathrm{FIR}) / M_{\mathrm{H}_{2}}$ and $S(60) / S(100)$ has been already found in a number of studies (e.g., Young et al. 1986a). The correlation found here is not as tight as that for $\Sigma(\mathrm{FIR})$ vs. $\log \Sigma\left(\mathrm{H}_{2}\right)$. The data points of both NGC 4038/4039 and IRAS 00057+4021 make the correlation worse though we do not understand why these data are far from the correlation for the remaining galaxies. Since the $L(\mathrm{FIR}) / M_{\mathrm{H}_{2}}$ ratio provides a measure of star formation efficiency (Young et al. 1986a, 1986b; S91), this correlation suggests that the higher star formation efficiency leads to the production of more heating photons per dust grain, resulting in the higher dust temperature.

Although S91 obtained a significant correlation between $\log L(\mathrm{FIR}) / M_{\mathrm{H}_{2}}$ vs. $\log \Sigma\left(\mathrm{H}_{2}\right)$ for 14 high-luminosity galaxies, we do not find this correlation for all the galaxies in S91 and for the galaxies in DS98. The little correlation between $L(\mathrm{FIR}) / M_{\mathrm{H}_{2}}$ and $\Sigma\left(\mathrm{H}_{2}\right)$ means that the star formation efficiency is not controlled by the surface gas density.

In Figure 2, we show diagrams of $\Sigma(\mathrm{FIR})$ and $\Sigma\left(\mathrm{H}_{2}\right)$ as a function of $A$ in logarithmic scales. It is shown that the two kinds of surface densities are almost inversely proportional to the surface, $A$; 1) $\Sigma(\mathrm{FIR}) \propto A^{a}: a=-0.77 \pm 0.15$ for the $\mathrm{S} 91$ sample, $-1.05 \pm 0.15$ for the DS98 sample, and $-0.85 \pm 0.13$ for the total sample, and 2) $\Sigma\left(\mathrm{H}_{2}\right) \propto A^{b}: b=-0.74 \pm 0.13$ for the S91 sample, $-0.90 \pm 0.09$ for the DS98 sample, and $-0.82 \pm 0.12$ for the total sample. The correlation coefficients for the total sample are -0.80 and -0.82 , respectively. Therefore, one important thing in the luminous starburst mergers is how the gas clouds are accumulated in smaller regions. We suggest that more concentrated gaseous systems tend to experience more intense starbursts such that $\Sigma(\mathrm{SFR}) \propto \Sigma($ gas $)$. 


\section{DISCUSSION}

We consider the physical meaning of the linear relationship between $\Sigma($ FIR $)$ and $\Sigma\left(\mathrm{H}_{2}\right)$. As mentioned before, this relationship suggests the star formation law of $\Sigma(\mathrm{SFR}) \propto \Sigma\left(\mathrm{H}_{2}\right)$. This kind of relation has been discussed by many authors in terms of so-called Schmidt law (Schmidt 1959); i.e., the star formation law remains the simple gas-density power law; i.e., $\Sigma(\mathrm{SFR}) \propto \Sigma(\text { gas })^{N}$. In the derivation of the Schmidt law, it is recommended to use the

total (i.e., $\mathrm{H} \mathrm{I}+\mathrm{H}_{2}$ ) gas mass density. However, since the nuclear gas in the high-luminosity starburst mergers is often dominated by molecular gas (Mirabel \& Sanders 1989; see also Sanders \& Mirabel 1996), we can neglect the contribution of H I gas (Kennicutt 1998); i.e., $\Sigma($ gas $) \simeq \Sigma\left(\mathrm{H}_{2}\right)$. Therefore, the linear correlation between $\Sigma(\mathrm{FIR})$ and $\Sigma\left(\mathrm{H}_{2}\right)$ suggests that the star formation in the luminous starburst mergers can be described by a Schmidt law with $N \simeq 1$.

For disks of normal galaxies, Kennicutt (1989) derived a relation of $N \simeq 1.3 \pm 0.3$ (see also, Buat, Deharveng, \& Donas 1989; Buat 1992; Bosseli 1994; Deharveng et al. 1994). Recently, Kennicutt (1998) derived a star formation law for a sample of 36 infrared-selected starburst galaxies; $N \simeq 1.40 \pm 0.13$. This Schmidt law is much steeper than that derived in this study. This seems as due to the difference in sampling between Kennicutt (1998) and ours. In his sample, a number of less luminous starburst galaxies (e.g., IC 342, NGC 6946, and so on) are included. Although these galaxies experience the moderate star forming activity, they are basically isolated ordinary spiral galaxies. On the other hand, our sample comprises mostly major mergers. In fact, the steeper Schmidt law derived by Kennicutt (1998) appears to be biased by the inclusion of the less-luminous, non-merger galaxies.

As mentioned in Section 1, there are three alternative mechanisms to initiate starbursts in merging galaxies; 1) the gravitational instability in nuclear gas disks (e.g., Kennicutt 1998 and references therein), 2) efficient cloud-cloud collision driven by violent gas motion 
in their central regions (e.g., Young et al. 1986a; S91), and 3) triggering through the dynamical disturbance driven by a supermassive binary (Taniguchi \& Wada 1996). A Schmidt law with $N \simeq 2$ can be achieved by the star formation driven by cloud-cloud collisions (Scoville, Sanders, \& Clemens 1986). On the other hand, a Schmidt law with $N \simeq 1-1.5$ implies the star formation triggered by the gravitational instability of gaseous disks (Kennicutt 1989, 1998; Larson 1988; Elmegreen 1994). The Schmidt law derived here (i.e., $N \simeq 1$ ) favors the gravitational instability scenario rather than the cloud-cloud collision model. Finally, we cannot rule out the third possibility because this model also adopts the gravitational instability formalism.

Recently, Taniguchi, Trentham, \& Shioya (1998) proposed a starburst-driven starburst mechanism in order to understand the formation of blue super star clusters in ULIGs such as Arp 220 (see also Shioya, Taniguchi, \& Trentham 1998). In such a mechanism the infalling dense gas disk is unstable gravitationally and collapses to form massive gaseous clumps. These clumps are exposed to the external high pressure from the superwind driven by the ongoing starburst at the very center of the galaxy. This external pressure leads to further collapse of the clumps which in turn leads to massive star formation in them. Our result is consistent with this scenario.

Starbursts in major mergers have been also studied by numerical simulations (e.g., Mihos \& Hernquist 1994 and references therein). In most the simulations with an SPH code, the star formation law is often assumed to follow a Schmidt law. For example, Mihos \& Hernquist (1994) adopted the Schmidt law with $N=1.5$. However, our result shows that the Schmidt law with $N \simeq 1$ describes the star formation in the actual high-luminosity major mergers. Therefore, we recommend that future numerical simulations for gas-rich

\footnotetext{
${ }^{2} \mathrm{~A}$ blast wave driven by a collective effect of a large number of supernovae in the very core of the galaxy (e.g., Heckman, Armus, \& Miley 1990; Heckman et al. 1996).
} 
major mergers will use the Schmidt law with $N \simeq 1$.

We would like to thank an anonymous referee for useful comments. Y.O. was supported by the Grant-in-Aid for JSPS Fellows by the Ministry of Education, Science, Sports and Culture. This work was supported in part by the Ministry of Education, Science, Sports and Culture in Japan under Grant Nos. 07055044, 10044052, and 10304013. 


\section{REFERENCES}

Barnes, J. E., \& Hernquist, L. E. 1991, ApJ, 370, L65

Buat, V. 1992, A\&A, 264, 444

Buat, V., Deharveng, J. M., \& Donas, J. 1989, A\&A, 223, 42

Bosseli, A. 1994, A\&A, 290, 1

Deharveng, J. M., Sasseen, T. P., Buat, V., Bowyer, S., Lampton, M., \& Wu, X. 1994, A\&A, 289, 715

de Vaucouleurs, G., de Vaucouleurs, A., Corwin, H. G., Jr., Buta, R. J., Paturel, G., \& Forqué, P. 1991, Third Reference Catalogue of Bright Galaxies (Springer-Verlag)

Downes, D., \& Solomon, P. M. 1998, ApJ, in press (DS98) astro-ph/9806377)

Elmegreen, B. G. 1994, ApJ, 425, L73

Genzel, R., et al. 1998, ApJ, 498, 579

Heckman, T. M., Armus, L., \& Miley, G. K. 1990, ApJS, 74, 833

Heckman, T. M., Dahlem, M., Eales, S. A., Fabbiano, G., \& Weaver, K. 1996, ApJ, 457, 616

Helou, G. I., Soifer, B. T., \& Rowan-Robinson, M. 1985, ApJ, 298, L7

Kennicutt, R. C. Jr. 1989, ApJ, 344, 685

Kennicutt, R. C. Jr. 1998, ApJ, 498, 541

Larson, R. B. 1988, in Galactic and Extragalactic Star Formation, ed. R. E. Pudritz \& M. Fich (Dordrecht: Kluwer), 435

Mirabel, I. F., \& Sanders, D. B. 1989, ApJ, 340, L53

Moshir, M., et al. 1992, Explanatory Supplement to the IRAS Faint Source Survey, Version 2, JPL-D-10015 8/92 (Pasadena: JPL) 
Mihos, C. J., \& Hernquist, L. 1994, ApJ, 431, L9

Sanders, D. B., et al. 1988, ApJ, 325, 74

Sanders, D. B., \& Mirabel, I. F. 1996, ARA \& A, 34, 749

Sanders, D. B., Scoville, N. Z., \& Soifer, B. T. 1991, ApJ, 370, 158

Schmidt, M. 1959, ApJ, 129, 243

Scoville, N. Z., Sanders, D. B., \& Clemens, D. P. 1986, ApJ, 310, L77

Scoville, N. Z., Sargent, A. I., Sanders, D. B., \& Soifer, B. T. 1991, ApJ, 366, L5 (S91)

Scoville, N. Z., Yun, M. S., \& Bryant, P. M. 1997, ApJ, 484, 702

Shaya, E., Dowling, D. M., Currie, D. G., Faber, S. M., \& Groth, E. J. 1994, AJ, 107, 1675

Shioya, Y., Taniguchi, Y., \& Trentham, N. 1998, ApJ, submitted

Soifer, B. T., et al. 1984, ApJ, 283, L1

Solomon, P. M., \& Sage, L. J. 1988, ApJ, 334, 613

Taniguchi, Y., Trentham, N., \& Shioya, Y. 1998, ApJ, 504, L79

Taniguchi, Y., \& Wada, K. 1996, ApJ, 469, 581

Wright, G. S., Joseph, R. D., \& Meikle, W. P. S. 1984, Nature, 309, 31

Young, J. S., Kenney, J. D., Tacconi, L., Claussen, M. J., Hunag, Y.-L., Tacconi-Garman, L., Xie, S., \& Schloerb, F. P. 1986a, ApJ, 311, L17

Young, J. S., Schloerb, F. P., Kenney, J. D., \& Lord, S. D. 1986b, ApJ, 304, 443 
Table 1. Properties of the sample of galaxies

\begin{tabular}{lcccccccccc}
\hline \hline & & & & & & & & & & \\
\multicolumn{1}{c}{$(1)$} & $(2)$ & $(3)$ & $(4)$ & $(5)$ & $(6)$ & $(7)$ & $(8)$ & $(9)$ & $(10)$ & $(11)$ \\
\hline \multicolumn{1}{c}{ Scoville et al. (1991) sample } & & & & & & & \\
\hline Mrk231 & $165^{b}$ & $<2.76$ & 32.0 & 30.3 & 1.06 & 12.08 & 10.51 & 1.57 & $>4.71$ & $>3.13$ \\
I17208 & $172^{d}$ & 1.28 & 31.1 & 34.9 & 0.89 & 12.13 & 10.73 & 1.40 & 5.42 & 4.02 \\
Arp220 & $74^{b}$ & 0.29 & 103.8 & 112.4 & 0.92 & 11.91 & 10.17 & 1.73 & 6.50 & 4.76 \\
VIIZw31 & $219^{d}$ & 2.67 & 5.6 & 9.6 & 0.58 & 11.66 & 10.46 & 1.20 & 4.30 & 3.11 \\
I10173a & $194^{c}$ & 3.27 & 5.8 & 5.5 & 1.06 & 11.48 & 9.95 & 1.53 & 3.96 & 2.42 \\
N6240 & $98^{b}$ & 1.65 & 22.7 & 27.8 & 0.82 & 11.52 & 10.03 & 1.49 & 4.58 & 3.09 \\
IC694 & $42^{c}$ & 0.30 & 103.7 & 107.4 & 0.97 & 11.41 & 9.59 & 1.82 & 5.96 & 4.14 \\
VV114 & $81^{c}$ & 0.93 & 22.6 & 30.4 & 0.74 & 11.35 & 10.03 & 1.33 & 4.92 & 3.59 \\
N1614 & $62^{b}$ & 0.60 & 32.3 & 32.7 & 0.99 & 11.25 & 9.78 & 1.47 & 5.19 & 3.72 \\
Arp55 & $160^{c}$ & 3.13 & 6.0 & 10.2 & 0.58 & 11.41 & 10.22 & 1.19 & 3.92 & 2.73 \\
N1068 & $14^{b}$ & 0.10 & 176.2 & 224.0 & 0.79 & 10.74 & 9.46 & 1.28 & 6.21 & 4.93 \\
N7469 & $67^{b}$ & 0.82 & 25.9 & 34.9 & 0.74 & 11.26 & 9.89 & 1.37 & 4.94 & 3.57 \\
Zw049.057 & $52^{c}$ & 0.40 & 20.8 & 29.4 & 0.71 & 10.95 & 9.67 & 1.28 & 5.24 & 3.96 \\
N828 & $73^{b}$ & 0.92 & 10.9 & 22.8 & 0.48 & 11.03 & 10.09 & 0.94 & 4.61 & 3.67 \\
N2146 & $17^{b}$ & 0.33 & 131.0 & 184.2 & 0.71 & 10.78 & 9.43 & 1.35 & 5.26 & 3.90 \\
N3079 & $20^{b}$ & 0.26 & 44.5 & 89.2 & 0.50 & 10.52 & 9.56 & 0.97 & 5.21 & 4.25 \\
N520 & $28^{b}$ & 0.38 & 30.9 & 45.8 & 0.67 & 10.58 & 9.47 & 1.11 & 4.92 & 3.80 \\
N4038/9 & $26^{b}$ & 0.49 & 39.5 & 72.3 & 0.55 & 10.65 & 9.07 & 1.58 & 4.78 & 3.20
\end{tabular}

Downes \& Solomon (1998) sample

\begin{tabular}{lllllllllll}
\hline $\mathrm{I} 00057^{\mathrm{a}}$ & $181^{d}$ & 0.48 & 4.5 & 4.3 & 1.04 & 11.31 & 10.27 & 1.04 & 5.44 & 4.40 \\
$\mathrm{I} 02483^{\mathrm{a}}$ & $207^{d}$ & 0.90 & 4.0 & 6.9 & 0.58 & 11.46 & 10.16 & 1.31 & 5.06 & 3.75
\end{tabular}


Fig. 1.- Diagrams of $\log L$ (FIR), $\log \Sigma(\mathrm{FIR})$, and $\log L(\mathrm{FIR}) / M_{\mathrm{H}_{2}}$ as functions of $\log$ $\Sigma\left(\mathrm{H}_{2}\right)$ and $\log S(60) / S(100)$. The OVRO sample is shown by filled circles while the IRAM one is shown by open circles. The filled squares for the four ULIGs observed by S91 (Arp 220, Mrk 231, VII Zw 31, and IRAS 17208-0014) show the data points when we adopt the sizes obtained by DS98. However, note that the data points of the four ULIGs in the $\log$ $\Sigma($ FIR $)$ vs. $\log S(60) / S(100)$ are the same as those for DS98.

Fig. 2.- Diagrams of $\log \Sigma(\mathrm{FIR})$ and $\log \Sigma\left(\mathrm{H}_{2}\right)$ as a function of the surface $A$. The symbols have the same meanings as those in Figure 1. 
Table 1-Continued

\begin{tabular}{ccccccccccc}
\hline \hline & & & & & & & & & & \\
$(1)$ & $(2)$ & $(3)$ & $(4)$ & $(5)$ & $(6)$ & $(7)$ & $(8)$ & $(9)$ & $(10)$ & $(11)$ \\
\hline VIIZw31 & $219^{d}$ & 1.27 & 5.6 & 9.6 & 0.58 & 11.66 & 10.70 & 0.96 & 4.95 & 3.99 \\
I10565 & $172^{d}$ & 0.67 & 12.1 & 15.1 & 0.80 & 11.73 & 10.38 & 1.35 & 5.59 & 4.24 \\
Mrk231 & $165^{b}$ & 0.36 & 32.0 & 30.3 & 1.06 & 12.08 & 10.35 & 1.73 & 6.47 & 4.74 \\
Arp193 & $92^{b}$ & 0.25 & 15.4 & 25.2 & 0.61 & 11.34 & 10.22 & 1.12 & 6.06 & 4.94 \\
Mrk273 & $152^{b}$ & 0.13 & 21.7 & 21.4 & 1.02 & 11.85 & 10.33 & 1.51 & 7.13 & 5.62 \\
Arp220 & $74^{b}$ & 0.16 & 103.8 & 112.4 & 0.92 & 11.91 & 10.43 & 1.48 & 7.00 & 5.52 \\
I17208 & $172^{d}$ & 0.75 & 31.1 & 34.9 & 0.89 & 12.13 & 10.67 & 1.46 & 5.88 & 4.42 \\
I23365 & $260^{d}$ & 0.63 & 7.1 & 8.4 & 0.85 & 11.85 & 10.39 & 1.46 & 5.75 & 4.29 \\
\hline
\end{tabular}

Note. - (1) Name, (2) Distance (Mpc); heliocentric velocities are taken from (b) de Vaucouleurs et al. (1991), (c) S91, and (d) DS98, (3) Radius (kpc), (4) S(60) (Jy), (5) $S(100)(\mathrm{Jy}),(6) S(60) / S(100),(7) \log L(\mathrm{FIR})\left(L_{\odot}\right),(8) \log M\left(\mathrm{H}_{2}\right)\left(M_{\odot}\right),(9) \log$ $L(\mathrm{FIR}) / M\left(\mathrm{H}_{2}\right)\left(L_{\odot} / M_{\odot}\right),(10) \log \Sigma(\mathrm{FIR})\left(L_{\odot} / \mathrm{pc}^{2}\right)$, and $(11) \log \Sigma\left(\mathrm{H}_{2}\right)\left(M_{\odot} / \mathrm{pc}^{2}\right)$ ${ }^{\mathrm{a}} \mathrm{I} 17208$ = IRAS 17208-0014; I10173 = IRAS 10173+0828; Zw049 = Zw049.057; I00057 = IRAS $00057+4021 ; \quad$ I02483 = IRAS $02483+4302 ; \quad$ I10565 = IRAS $10565+2448 ;$ I $23365=$ IRAS $23365+3604$. 


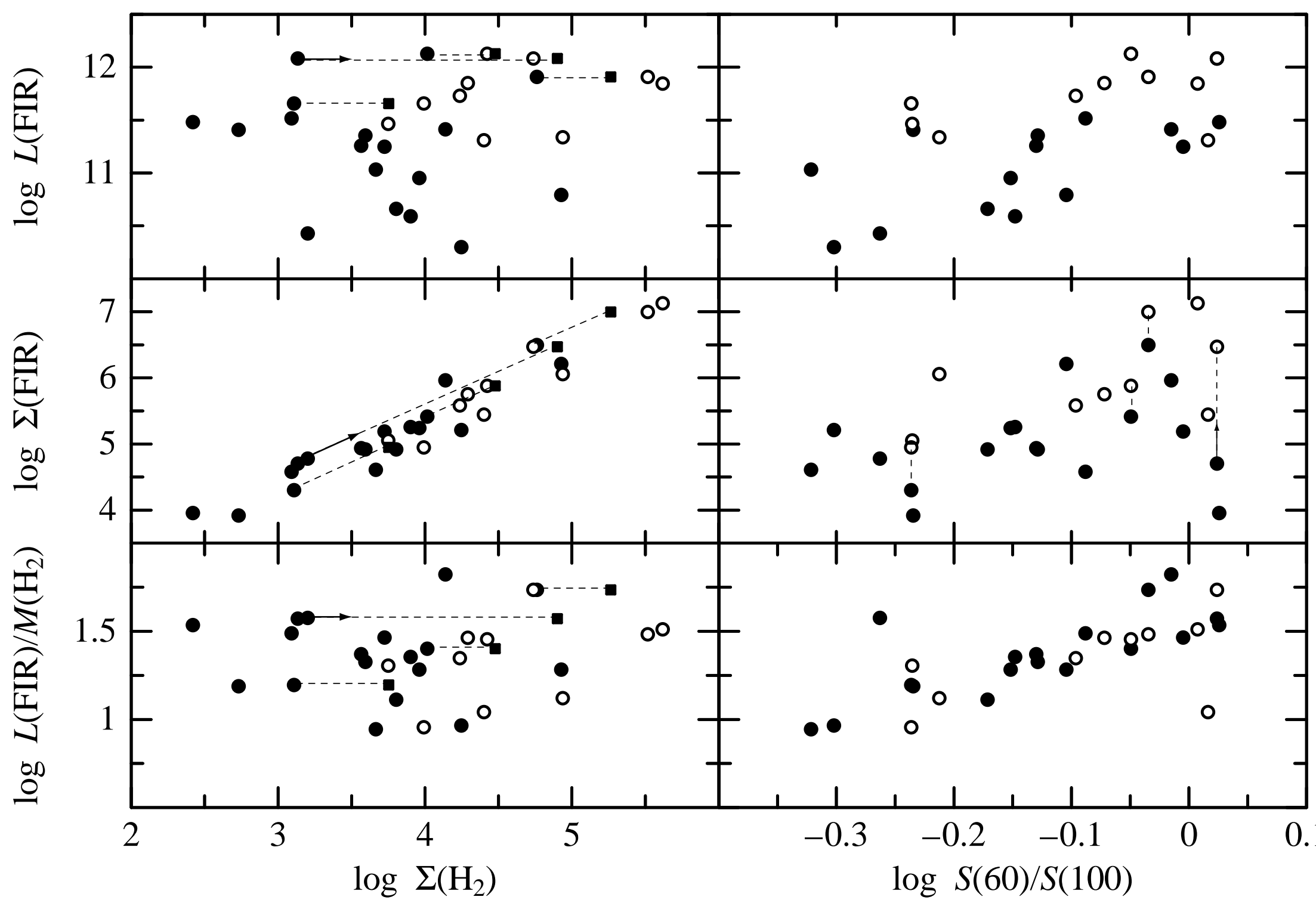




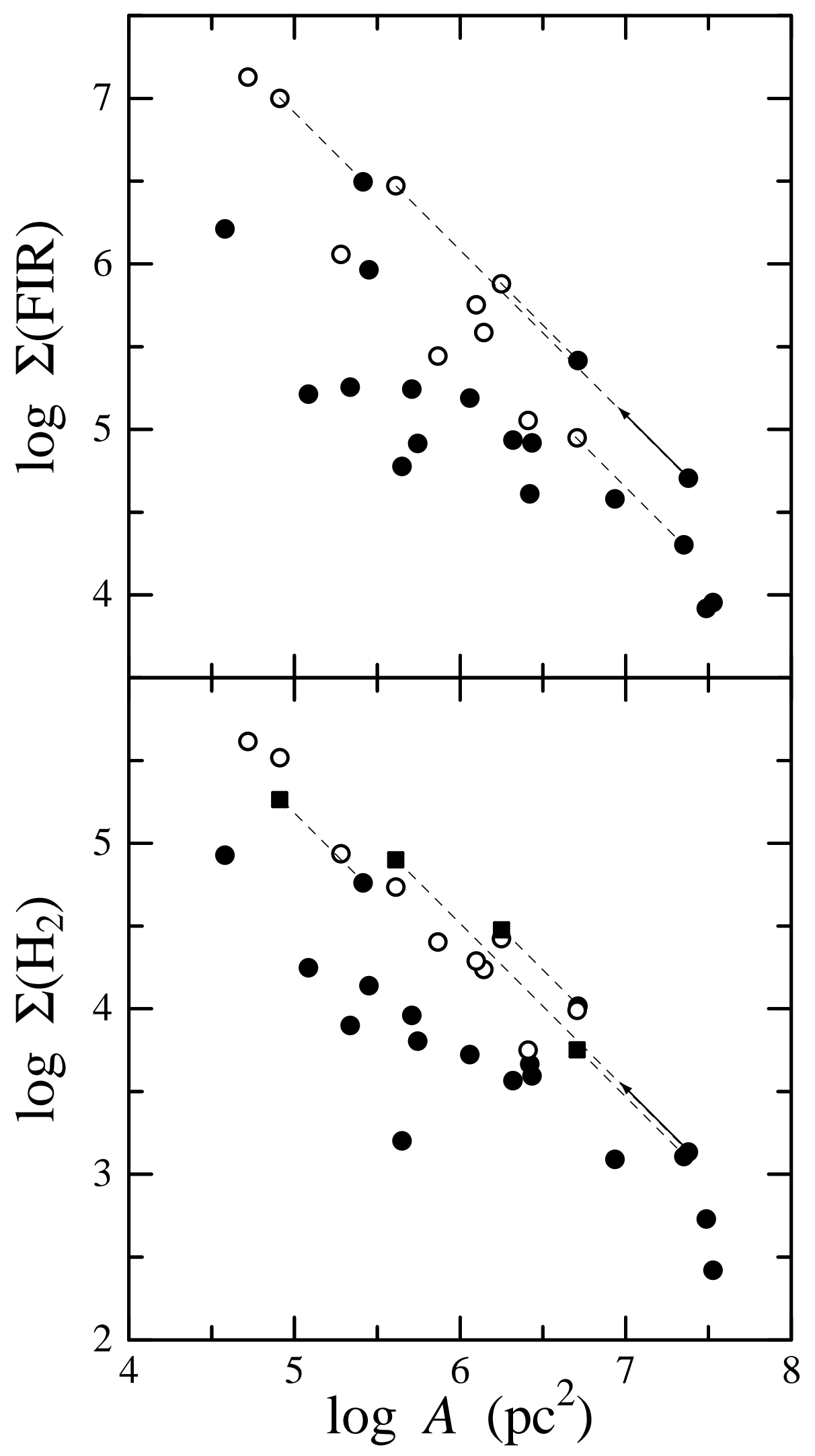

